\title{
Craniofaciofrontodigital syndrome
}

INSERM

\section{Source}

INSERM. (1999). Orphanet: an online rare disease and orphan drug data base.

Craniofaciofrontodigital syndrome. ORPHA:363705

Craniofaciofrontodigital syndrome is a rare multiple congenital anomalies syndrome characterized by mild intellectual disability, short stature, cardiac anomalies, mild dysmorphic features (macrocephaly, prominent forehead, hypertelorism, exophthalmos), cutis laxa, joint hyperlaxity, wrinkled palms and soles and skeletal anomalies (sella turcica, wide ribs and small vertebral bodies). 